\title{
ERFUNDENE TRADITION. VOLKSLIEDER ALS NATIONALE STEREOTYPEN
}

\author{
Eckhard John
}

Volkslieder werden gemacht - und immer wieder neu entdeckt. In letzter Zeit haben Volkslieder erneut Konjunktur. Dabei ist eine Vielfalt von Produktionen entstanden, die durch ihre innovativen Ansätze aufhorchen lassen. Diese Versuche, traditionelle Lieder auf produktive Weise neu zu bearbeiten, stehen in deutlichem Kontrast zum altbackenen Kulturpessimismus, der mit Volksliedern herkömmlicherweise häufig einhergeht (Credo: "Keiner singt mehr - Refrain: »Rettet unsere Lieder «). Doch werden auch diese musikalisch auf der Höhe unserer Zeit angesiedelten Projekte bisweilen als neuer Sound of Heimat gehandelt. ${ }^{1}$ Dies mag marktstrategisch eine prägnante Etikettierung sein, gleichwohl stellt sich die Frage, in wie weit solche Verortung für die Sache selbst adäquat und hilfreich ist. In der Popularmusikforschung spielte der Bereich einer potentiellen German roots music in jüngerer Zeit keine nennenswerte Rolle mehr. Die thematischen Schwerpunktsetzungen haben sich hier grosso modo in eine gänzlich andere Richtung entwickelt: Populäre Musik aus Zeiten vor der Erfindung des Rock'n' Roll oder des Grammophons fand im einschlägigen Forschungsdiskurs immer weniger Berücksichtigung. Volkslieder galten bei der Generation der Heinound Musikantenstadl-Traumatisierten ohnehin meist als ebenso peinlich wie reaktionär. ${ }^{2}$ Doch wenn das Motto "Typisch deutsch « auf der Agenda der Popularmusikforschung steht, dann darf das Thema Volkslied natürlich nicht fehlen.

1 Siehe beispielsweise Sound of Heimat. Deutschland singt (2012, Regie: Arne Birkenstock und Jan Tengeler).

2 Dabei werden die verschiedenen kritischen Auseinandersetzungen mit dem Volkslied seit den 1960er Jahren nicht übersehen. Erinnert sei in diesem Zusammenhang aber auch an Köpfe wie Dieter Hildebrandt, der wenige Tage vor der Gießener ASPM-Tagung im November 2013 gestorben ist. Seine Version von »Der Mond ist aufgegangen « ist auch als unmittelbare Referenz an das Tagungsmotto lesbar; siehe nachfolgende Seite. 


\section{Der Mond ist aufgegangen}

Helmut Kohl spricht Matthias Claudius:

\section{Der Mond,}

meine Damen und Herren, und das möchte ich hier in aller Offenheit sagen, ist aufgegangen!

Und niemand von Ihnen, liebe Freunde, meine Damen und Herren, wird mich daran hindern, hier in aller Entschlossenheit festzustellen:

\section{Die goldnen Sternlein prangen}

und wenn Sie mich fragen, meine Freunde, wo, dann sage ich es Ihnen:

\section{am Himmel!}

Und zwar, und das sei hier in aller Eindeutigkeit gesagt, so, wie meine Freunde und ich uns immer zu allen Problemen geäußert haben:

\section{hell und klar.}

Und ich scheue mich auch nicht, hier an dieser Stelle ganz konkret zu behaupten:

\section{Der Wald steht schwarz und...}

lassen sie mich das hinzufügen

und schweiget.

Und hier sind wir doch alle aufgerufen - gemeinsam - , die uns alle tiefbewegende Frage an uns gemeinsam zu richten: Wie geht es denn weiter? Und ich habe den Mut und die tiefe Bereitschaft und die Entschlossenheit, hier in allem Freimut und aller Entschiedenheit zu bekennen, dass ich es weiß!

Nämlich:

\section{Und aus den Wiesen steiget}

das, was meine Reden immer ausgezeichnet hat:

der weiße Nebel wunderbar.

Dieter Hildebrandt ${ }^{3}$

3 Zit. nach der Fassung auf Dieter Hildebrandts Homepage; siehe http://www. dieterhildebrandt.com/texte_02.php. Dort mit folgender Anmerkung: »Kohl hatte die amüsierende Manie, den vor ihm liegenden Text mit eigenen Ideen anzureichern. Sehr zum Entsetzen des jeweiligen Autors. Da er auf Stil und Aussage eines Textes gewöhnlich so gut wie keine Rücksicht nahm, kam ich auf die Idee, ihm einen klassischen, von ihm verehrten Text auf das Rednerpult zu legen und mir vorzustellen, wie er mit diesem wohl umgehen würde. « - Wie alle »Volkslieder « lebt auch dieser Text primär von seiner »mündlichen Tradierung «, wobei die verschiedenen Hildebrandtschen »Varianten« unschwer im medialen Gedächtnis der Videokanäle (wie YouTube) zu finden sind. 
Mit der Frage, welche Deutschheiten oder Deutschtümer sich mit diesen Liedern verbinden, gehen freilich wenig Hoffnungen auf erhellende Erkenntnisse einher, vielmehr beklemmende Erinnerungen an »finstere Zeiten « (Bert Brecht). Volkslieder fungierten ehedem als zentrales Paradigma einer Popularmusikforschung, die als »musikalische Volkskunde« firmierte. Innen wurde ein besonderer historiographischer Stellenwert zugesprochen: Volkslieder galten als eine Art national-musikalische Ursuppe, als Keimzelle der deutschen Musik und Wurzel einer deutschen Musikgeschichte. Modellhafte Ausprägungen dieser Sichtweise hatten zu Zeiten der NS-Herrschaft unter dem Label »Vom Deutschtum in der Musik« ihre Wirkmächtigkeit entfalten können, als Musikwissenschaftler vom Schlage eines Joseph Müller-Blattau ein biologistisch-organisches Musikgeschichtsbild entwarfen und dieses mit der Metapher des Lebensbaumes beschrieben: dabei bildete die Volksmusik Wurzeln und Stamm, während die prächtige, ausladende Krone der Kunstmusik entsprach (vgl. John 1991: 172). Auch wenn offensichtlich war, wie unverblümt nazistische Ideologie in diesem Sinnbild das Blut aus dem Boden in die Musik pumpte, prägten solche Sichtweisen Musikwissenschaft und Popularliedforschung noch Jahrzehnte nach 1945.

Heute ist die Situation eine andere. Die einstmals völkische Volkskunde hat sich längst zu einer modernen Kulturwissenschaft entwickelt und Hermann Bausinger - einer der Protagonisten dieses Wandels - veranschaulicht in seiner Studie Typisch deutsch eindrücklich, wie sehr »an der Gültigkeit von Aussagen über das Typische Abstriche zu machen« (Bausinger 2000: 19) sind. Denn »es sind immer nur Annäherungen, Konstruktionen einer komplexen und nicht präzise faßbaren Realität « (ebd.). Bausinger macht deutlich, dass es sich bei solchen Stereotypen in erster Linie um Zuschreibungen handelt, die häufig auf einer Kontrasterfahrung beruhen. An den Gedanken, dass Typisierungen immer mit Bestrebungen nach Abgrenzungen und Distinktion einhergehen, möchte ich im Folgenden anknüpfen. So wird etwa in einem jüngeren jüdischen Witz die Frage aufgeworfen: »Was ist der Unterschied zwischen einem Juden und einem Deutschen?« Die Antwort auf diese elementare Frage ist simpel: »Der Deutsche mag Klesmer « - das also wäre »typisch deutsch«! So gesehen läge es nahe, an dieser Stelle den in Deutschland besonders ausgeprägten Klesmerboom der letzten Jahrzehnte nachzuzeichnen und eingehend zu untersuchen. ${ }^{4}$ Es mag als eine Art Treppenwitz

4 Damit ließe sich ohne weiteres ein eigener Beitrag bestreiten; und dieser würde zur Thematik »Typisch deutsch « vermutlich noch so manchen aufschlussreichen Gesichtspunkt beisteuern. Es ist ohnehin bemerkenswert, dass sich auf einer -Tagung wie dieser k kein einziger Beitrag mit dem Aspekt jüdischer Lebenswelten hinsichtlich der populären Musik in Deutschland beschäftigt hat. Diese Absenz könnte man ihrerseits wiederum als typisch deutsch ansehen. 
der Geschichte erscheinen, dass ausgerechnet in jenem Land, das für die millionenfache Ermordung der Juden in Europa verantwortlich ist, das Klesmer-Revival auf größte Resonanz stößt - während just diejenigen, als deren vermeintliche roots music Klesmer gilt, dessen Erfolgswelle häufig mit Skepsis und Distanz gegenüberstehen. Dass die erwartbare Ordnung und Zuordnung volksmusikalischer Traditionen hier einfach auf den Kopf gestellt wird, generiert die Pointe. Der Witz an der Sache ist aber letztlich, dass dies auf der stillschweigenden Übereinkunft beruht, gruppenspezifische Identitäten würden sich in ethnisch codierten Formen traditioneller Musik artikulieren und abbilden. Dies ist auch der Punkt, in dem sich der jüdische Witz mit dem deutschen Volkslied trifft - genauer: mit dem erwähnten Umstand, dass das Volkslied unweigerlich auf der Tagesordnung steht, wenn Fragen nach dem typisch Deutschen in der Popularmusik aufgeworfen werden.

Prinzipielle Überlegungen dazu - was ist typisch? was ist deutsch? werden an anderer Stelle erörtert. ${ }^{5}$ Hier ist indes eine weitere grundlegende Frage unvermeidbar: Was ist eigentlich ein Volkslied? Diese Frage stellt sich auch jenseits akademischer Reflexion. Was ist beispielsweise gemeint, wenn eine Musikerin wie Sophie Hunger sich im Titelsong ihres zweiten Albums wünscht: »Komm, bitte sing mir ein Volkslied $\aleph^{6}$ - ein Verlangen, das durch refrainartige Wiederholung noch besonderes Gewicht und Profil gewinnt? Inhaltlich ist diese Textzeile schon aufgrund ihrer bloßen Existenz bemerkenswert: Wer hätte vor zehn oder zwanzig Jahren in einem deutschen Rock- oder Pop-Song ernsthaft den Wunsch artikuliert: »Bitte sing mir ein Volkslied « - das wäre allenfalls als Parodie oder Satire denkbar gewesen. Hier deutet sich an, dass sich bei der jüngeren Musikergeneration das Verhältnis zum »Volkslied « offenbar verändert hat.

Andererseits gibt der Text von Hungers Song »1983 «, aus dem die exponierte Zeile stammt, kaum Auskunft darüber, an was für Lieder sie dabei eigentlich denkt. Gesagt wird lediglich, dass es Volkslieder »nicht mehr gibt«. Somit bleibt weitgehend offen, was mit dem erbetenen Volkslied überhaupt gemeint ist, zumal die Bandbreite möglicher Bezüge denkbar weit gestreut ist: ein besonders altes Lied, ein mündlich tradiertes, eines aus bäuerlich-ländlichem Milieu, eine Vertonung Gustav Mahlers aus Des Knaben Wunderhorn, ein Lied, das ihre Großmutter in Kindertagen sang, eines, das Erinnerungen weckt: an Jugendgruppen und Zeltlager, an quälende Schulstunden, an Heino-Platten und »Volksmusik«-Shows - oder an die Folkbewegung, an die »anderen Lieder« der kleinen Leute und die »demo-

5 Neben Bausinger siehe etwa die Beiträge von Thomas Phleps und Ekkehard Jost im vorliegenden Band.

6 Sophie Hunger (2010). »1983. «Auf: 1983. Two Gentlemen Records, twogtl 009-2. 
kratischen Lieder « des sozialen Widerstands - die Assoziationsfelder ließen sich unschwer erweitern. Doch die angeführten reichen bereits aus, um den Klärungsbedarf zu illustrieren: Was also ist ein Volkslied?

Generationen von Forschern, von Wissenschaftlern und Enthusiasten, haben versucht, plausible Antworten auf diese Frage zu finden; sie haben nach den Ur-sprüngen und Ur-Texten gesucht; haben das Volkslied als neues poetisches Ideal auf den Schild gehoben - oder im »Volkston« ein damit korrespondierendes musikalisches Signum kreiert; haben das Volkslied zum Leitbild romantischer Ästhetik zurechtgeschrieben - oder mit philologischer Akribie traditionelle Lieder aus mündlicher Überlieferung aufgezeichnet; haben es als Ausdruck der schöpferischen Produktivkraft eines »Volkes« beschworen - oder als ästhetische Codierung einer vermeintlichen »Volksseele«; haben es im Sinne der frühen Volkskunde als konservatives Element im ländlich-bäuerlichen Milieu verortet - oder mit Wolfgang Steinitz als ein sozialkritisches, politisch-oppositionelles Medium interpretiert; haben es zum wolkigen Sinnbild sozialer Gemeinschaftsideologie stilisiert - oder mit dem nüchternen Blick eines John Meier als ein spezifisches Rezeptionsphänomen bewertet; haben es zu einem nationalen Symbol überhöht und mit völkischem Pathos überfrachtet; haben es als so genannte »Grundschicht « kreativen Schaffens in musikanthropologische Sichtweisen eingebaut - oder als rassenideologisches Instrument dem nazistischen Terror dienstbar gemacht.

Nicht zuletzt deshalb war die Haltung gegenüber dem Volkslied im 20. Jahrhundert stets auch eine gebrochene. Die eher überschwänglichnaive Volkslied-Euphorie des 19. Jahrhunderts wurde in den zurückliegenden hundert Jahren abgelöst durch ein höchst ambivalentes Verhältnis. Und das setzte nicht erst nach 1945, sondern bereits nach dem Ersten Weltkrieg ein. Kritische Intellektuelle mokierten sich damals gerne über die ästhetische Niedlichkeit oder den politisch gefährlichen Nationalismus zahlreicher Lieder. So auch der Schriftsteller Erich Weinert, der sich seinen eigenen Reim machte auf das deutsche »Einheitsvolkslied« (zit. nach Weinert 1970: 165):

»Stimmt an mit hellem, hohem Klang!

Nun muß sich alles wenden.

Wer nicht liebt Wein, Weib und Gesang

Mit Herzen, Mund und Händen.

Das Wandern ist des Müllers Lust.

Was blasen die Trompeten?

Wir treten mutig Brust an Brust

Zum Beten, ja zum Beten.

[...] 
Die Lerche schmettert himmelan.

Es geht von Mund zu Munde.

Der Kaiser ist ein lieber Mann

In einem kühlen Grunde.«

Dieser Text aus dem Jahr 1924 - eine Collage prominenter Zeilen aus beliebten Volksliedern des 19. Jahrhunderts - illustriert, dass nicht erst in den Zeiten tümlicher »Volksmusik«-Shows eine deutliche Skepsis gegenüber dem als Volkslied geltenden Repertoire vorhanden war. Und nach den bitteren Erfahrungen unter nationalsozialistischer Herrschaft hatte das Volkslied bei kritischen Geistern endgültig ausgespielt. Der Liedermacher Franz Josef Degenhard brachte 1968 in seinem Chanson »Die alten Lieder « eine verbreitete Haltung treffend auf den Punkt (hier zit. nach Degenhard 2006: 71):

»Tot sind unsre Lieder, unsre alten Lieder.

Lehrer haben sie zerbissen, Kurzbehoste sie verklampft, braune Horden totgeschrien,

Stiefel in den Dreck gestampft.«

Dennoch prägen Volkslieder die Kultur bis in unsere Tage, und zwar nicht nur in Formen politischer Indienstnahme, volkstümlicher Pflege, touristischer Folklorisierung oder kulturindustrieller Kommerzialisierung. Und gerade in den letzten zehn Jahren hat sich wieder ein neues Interesse an Volksliedern herauskristallisiert. Das Verblüffende und wirklich Neue daran ist, dass es vermehrt Musiker aus den verschiedensten Bereichen von Pop-, Rock- oder Jazz-Szene sind, die nunmehr auch traditionelle Lieder spielen. Die langjährige Kontaminierung des Volksliedes mit braun-schwarzem Muff und schlechtem Geschmack spielt für diese jüngere Musikergeneration offenkundig keine abschreckende Rolle mehr. Sie suchen erfreulich unverkrampft und experimentierfreudig nach musikalischen roots der Sorte Made in Germany - und finden sie in alten Liedern.

Die weitreichende historische Wirkungsmacht des Volksliedes wird verständlich, wenn man sich bewusst macht, dass die Volkslied-Idee wohl die mit Abstand erfolgreichste Innovation im Bereich der Popularmusik war, welche die Musikgeschichte des 19. Jahrhunderts maßgeblich beeinflusste. Parallel zur Etablierung der Idee einer absoluten Musik als autonomer Kunst war die Erfindung des Volkslieds die wohl weitreichendste Neuerung, die eine enorme Diskursmächtigkeit entwickelte und sich in opulenten Volkslied-Sammlungen und wissenschaftlichen Editionen manifestierte. Diese prägten das kulturelle Gedächtnis nachhaltig. Dabei avancierte der Deut- 
sche Liederhort von Ludwig Erk und Franz Magnus Böhme (1893/94) wie kein anderes Werk zur Kanon bildenden Instanz. Solche Popularlied-Editionen des ausgehenden 19. Jahrhunderts sind das Pendant zu den >Denkmälern< der musikalischen Hochkultur, mit denen die Tonkunst etwa zur gleichen Zeit ausstaffiert wurde, sie sind - wenn man so will - die entsprechenden >Monumente $<$. Die monumentalste wissenschaftliche Edition traditioneller Lieder aus Deutschland sollte die Publikation Deutsche Volkslieder mit ihren Melodien werden, zu deren Realisierung 1914 eigens ein Deutsches Volksliedarchiv gegründet wurde (vgl. John 2010a).

Für die Geschichte der Volkslied-Idee scheint mir das Konzept der Erfindung einer Tradition (vgl. Hobsbawn 1983) ein hilfreicher Ansatz für Verständnis und Analyse zu sein, zumal in Hinblick auf die sozialen Bezüge und Wechselwirkungen dieser Entwicklung. Die Etablierung des Volkslied-Gedankens war eng verwoben mit den aufkeimenden National-Ideen, mit den Prozessen des nation building, des Nationalismus und Chauvinismus - bis hin zu völkischer Ideologie und nazistischer Terrorherrschaft. Volkslied-Diskurse waren somit immer auch zutiefst verflochten mit nationalstaatlichen Ambitionen und nationalistischer Propaganda, und es mangelte nicht an entsprechenden Akteuren, Gruppen und Institutionen, die hier als einflussreiche Vermittlungsinstanzen auftraten: die Männergesangvereine als Wiege des »singenden deutschen Mannes « (Klenke 1998), die studentischen Burschenschaften sowie die Jugendbewegung des 20. Jahrhunderts. Hinzu kam das Militär und - nicht zuletzt - die Schule (von der Kriegserziehung im Kaiserreich über die jugendbewegte Musikpädagogik bis hin zur nazistischen »Volksgemeinschafts«-Phraseologie).

In diesen Netzwerken der Pädagogisierung und Popularisierung wurde ein kulturelles Gedächtnis für Volkslieder modelliert, das auf der Ebene der Zuschreibungen, Konstruktionen, Indienstnahmen, Ideologisierung und Politisierung eng mit den National- und Nationalismus-Diskursen verknüpft war. Aber es wäre zu kurz gegriffen, das Volkslied auf eine Rolle als musikalischer Steigbügelhalter des deutschen Nationalismus zu reduzieren. Zu vielschichtig sind - zum einen - die Gründe und Ursachen seines Erfolgs (und es ist just diese Vielschichtigkeit, auch seine Uneindeutigkeit, die Grundlage dieser Erfolgsgeschichte war). Zum anderen sollte man - bei aller DiskursKritik und -Analyse - die Ebene der Lieder selbst nicht aus den Augen verlieren. Denn hier lässt sich weit weniger eindeutig sagen, was daran »typisch deutsch « sein könnte. Es lassen sich schwerlich überzeugende Kriterien festmachen, die belegen würden, was an diesen Liedern - an Form, Struktur oder Inhalt - so besonders oder spezifisch »deutsch « sei. Ein Kriterium freilich gibt es, und dieses ist ebenso grundlegend wie banal: die deut- 
sche Sprache (samt den deutschsprachigen Mundarten) - das ist eo ipso »typisch deutsch«. Aber darüber hinaus sehe ich eigentlich nichts, was man als »typisch deutsche « Wesensmerkmale bei traditionellen Liedern dingfest machen könnte.

Weit stärker jedoch als die sprachlichen und musikalischen Texte konstituieren oftmals die soziokulturellen Kontexte eines Liedes dessen Bedeutungsebenen. Und in dieser Hinsicht ist nicht zu übersehen, dass die Geschichte einzelner Lieder und die damit einhergehenden Rezeptionsformen von den genannten Deutschtums-Diskursen durchsetzt und maßgeblich beeinflusst sind. Wie nachhaltig die jeweiligen Volkslied-Konzepte und Sammlungsintentionen die Rezeption eines Liedes beeinflussen können, lässt sich anhand einer eingehenden Rekonstruktion seiner jeweiligen Geschichte ablesen. Untersucht man die Rezeptionsgeschichte einzelner Lieder aus dem Kontext des sogenannten Volkslied-Repertoires, so lässt sich immer wieder beobachten, wie sie im Verlauf des historischen Prozesses mit jeweils spezifischen Legenden ausgestattet werden: Legenden etwa über Alter oder Herkunft oder Funktion eines Liedes. Diese Zuschreibungen formen die Geschichte und Bedeutung des jeweiligen Liedes meist im Sinne der Volkslied-Idee und machen sie in diesem Kontext dienstbar. Solche liedspezifischen Legendbildungen in ihrem historischen Kontext transparent zu machen, ist einer der grundlegenden Ansatzpunkte des Historisch-kritischen Liederlexikons, das wir im Deutschen Volksliedarchiv seit 2007 als frei zugängliche Online-Publikation (www.liederlexikon.de) aufgebaut haben. ${ }^{7}$ Das zentrale Forschungsprojekt widmete sich der Rezeptionsgeschichte »Traditioneller Lieder im 20. Jahrhundert . $^{8}$ Anhand dieses Liedrepertoires lässt sich die erwähnte Legendenbildung zu einzelnen Liedern paradigmatisch veranschaulichen. Offenbar brauchen solche Lieder spezifische Zuschreibungen, um als Volkslieder funktionieren zu können.

Dies sei am Beispiel der Ballade von den zwei Königskindern etwas näher dargestellt. Diese Ballade gilt landläufig als ein »klassisches Beispiel für ein altüberliefertes Lied; die Tradierung reicht kontinuierlich vom 15 . bis ins 20. Jahrhundert « ${ }^{9}$ - diese Sichtweise ist zweifellos typisch dafür, was man im Allgemeinen mit einem »echten Volkslied« verbindet: es sei sehr alt und seine Tradierung reiche »kontinuierlich « vom ausgehenden Mittelalter bis in unsere Gegenwart. Schaut man sich die konkreten Quellen und die Überlie-

7 Bis 2013 konnten dafür 275 Lieder mit über 1.200 Editionen bearbeitet werden; zur Konzeption siehe John 2010b.

8 Siehe http://www.liederlexikon.de/ueber_liederlexikon_de/projekte/dfg_proj ekt (letzter Zugriff am 27.5.2014).

9 So formuliert beispielsweise der entsprechende Wikipedia-Artikel, https://de. wikipedia.org/wiki/Es_waren_zwei_Königskinder (letzter Zugriff am 27.5.2014). 
ferungsgeschichte dieser Ballade jedoch genauer an, so ergibt sich ein anderes Bild: Denn das Lied ist vor dem Aufkommen der Volkslied-Idee lediglich in zwei Flugschriften aus der Zeit um 1580 überliefert. Eine allgemeine, volksliedartige Verbreitung im ausgehenden 16. Jahrhundert ist somit eher unwahrscheinlich; Belege aus dem Mittelalter fehlen völlig. Sicher ist eigentlich nur, dass man nichts Genaues weiß: Es ist gänzlich unbekannt, wer diese Ballade wo, wann und warum geschrieben, gesungen und verbreitet hat, auch über die musikalische Seite des Liedes ist nichts überliefert. Aber wir wissen, dass der Liedtext im 16. Jahrhundert noch gar keine »Königskinder « als Protagonisten hatte. Vielmehr lautete der Liedanfang damals:

»Zwischen zweyen burgen /

da ist ein tieffer See /

auff der einen burge /

da sitzet ein edler Herr.

Auff der andern burge /

do wont ein Junckfraw fein /

sie weren gern zusammen /

ach Gott möcht es gesein. «10

Weitere Spuren zu dieser Ballade gibt es erst 200 Jahre später, im Zuge der mit Herder einsetzenden Volkslied-Begeisterung und -Sammlung. Seit Beginn des 19. Jahrhunderts erscheint das Lied in entsprechenden Ausgaben, erhielt in dieser Zeit auch jene Melodie, die wir noch heute dazu kennen, ebenso die bekannten Eingangsverse:

»Es waren zwei Königskinder,

die hatten einander so lieb,

sie konnten beisammen nicht kommen,

das Wasser war viel zu tief.«11

Erst ab der zweiten Hälfte des 19. Jahrhunderts erschien das Lied dann vermehrt in Gebrauchsliederbüchern. Von einer »kontinuierlichen« Überlieferung (vom Mittelalter bis in die Gegenwart) kann somit ernstlich keine Rede sein. Doch im Verlauf des 19. Jahrhunderts wurde die Ballade von den Königskindern nun tatsächlich zu einem Lied mit äußerst hohem Verbreitungs-

10 Ein Hüpsch New Lied / zwischen zweyen burgen / da ist ein tieffer See / etc. [...]. Gedruckt zu Nürnberg / bey Valentin Fuhrman [um 1580], Str. 1 und 2 (von insgesamt 20); zit. nach der Edition im Historisch-kritischen Liederlexikon, http://www.liederlexikon.de/lieder/es_waren_zwei_koenigskinder, Edition A (letzter Zugriff am 27.5.2014).

11 Liederbuch für deutsche Künstler. Berlin: Vereins-Buchhandlung 1833; zit. nach der Edition im Historisch-kritischen Liederlexikon, URL s. Fußnote 10, Edition D. 
grad und großer Variantenbildung, wie man an zahllosen Aufzeichnungen aus mündlicher Überlieferung im 19. und 20. Jahrhundert ablesen kann. ${ }^{12}$

Wie aber wurde diese populäre Ballade nun zu einem »altüberlieferten Volkslied« mit vermeintlich mittelalterlichen Wurzeln, wie dies die herkömmliche Volksliedforschung postulierte? Den Brückenschlag dazu fand man in der Kombination der Königskinder-Ballade mit einem anderen Lied des 16. Jahrhunderts: »Ach Elslein, liebes Elslein mein«. Das Einzige, was beide Lieder gemeinsam hatten, war die Metapher vom Wasser als trennendem Element:

»Ach Elslein, liebes Elselein mein, wie gern wär' ich bei dir!

So sein zwei tiefe Wasser wohl zwischen dir und mir.«13

Das reichte früheren Forschern aus, um beide Lieder miteinander in Verbindung zu setzen (auch wenn die Wasser-Metapher in den beiden Texten unterschiedlich verwendet wurde). Denn das »Elslein«-Lied war im Banne der Volkslied-Idee aus mehreren Gründen eminent reizvoll: Dieses Lied war im 16. Jahrhundert tatsächlich recht verbreitet und zwar in durchaus unterschiedlichen Gestalten; und es fand sich dazu etwas, was die Ballade nicht hatte: Musik! Genau besehen sind sogar fast nur musikalische Quellen zu diesem Lied überliefert ${ }^{14}$ und die früheste davon stammt aus dem Glogauer Liederbuch, einer Handschrift aus der Zeit um 1480. Dieser »Elslein«-Beleg wurde wiederum zur Rückdatierung der Ballade benutzt und durch die konstruierte Kombination des »Elslein«-Liedes mit der »Königskinder «-Ballade konnte auch deren Herkunft ins ausgehende Mittelalter verlegt werden.

Noch abenteuerlicher wird es, wenn man sich vergegenwärtigt, wie aus diesem vermeintlich altüberlieferten Volkslied ein »deutsches« Volkslied gemacht wurde. Denn der Balladenstoff hatte seinen Ursprung unmissverständlich in der griechischen Antike: in der Sage von Hero und Leander, wie sie von Ovid und Musaios überliefert wurde. Er war schon im 16. Jahrhundert in anderen Ländern, zumal in den Niederlanden und in Skandinavien, in ähnlicher Weise wie im deutschen Sprachraum als Ballade präsent. Die Eindeutschung dieses Liedes spielte sich auf verschiedenen Ebenen ab, wobei

12 Ausführlich dazu Eckhard John (2013). »Es waren zwei Königskinder.« In: Historisch-kritisches Liederlexikon, URL s. Fußnote 10.

13 Hans Ott: Hundert vnd ainundzweintzig newe Lieder (Nürnberg 1534); zit. nach der Edition im Historisch-kritischen Liederlexikon, http://www.liederlexikon. de/lieder/ach_elslein_liebes_elselein, Edition A (letzter Zugriff am 27.5.2014).

14 Vgl. im Einzelnen Eckhard John (2013). »Ach Elslein, liebes Elselein.« In: Historisch-kritisches Liederlexikon, URL s. Fußnote 13. 
hier von dem recht komplexen Prozess nur einige Grundmuster skizziert werden können: Angesichts der Verbreitung der Ballade in anderen Ländern wurde zunächst eine Priorität der Entstehung in Deutschland postuliert und diese damit erklärt, dass sich die Ballade von Süden (Griechenland) über Deutschland nach Norden (Skandinavien) verbreitet habe; die umfangreichere frühe Überlieferung der Ballade in den Niederlanden (ab dem 16. Jahrhundert) wurde wiederum als Stellvertreter für eine angeblich verloren gegangene Tradierung in Deutschland interpretiert; und im 19. Jahrhundert wurde die mündliche Überlieferung in niederdeutscher (plattdeutscher) Mundart in den gedruckten Volkslied-Sammlungen und Liederbüchern meist in hochdeutsche Liedfassungen transformiert. Der ideologische Schulterschluss zu germanischen Vorzeiten, deren vermeintliches Erbe noch in dieser Ballade enthalten sei, wurde schließlich im Zuge völkisch-nazistischer Kontexte entwickelt und leitete sich einzig von jener »Nonne«ab, von der meist in der dritten Strophe berichtet wird (vgl. Fußnote 11):

»Da saß eine falsche Nonne,

Die that, als wenn sie schlief;

Sie thät die Kerzen auslöschen

Der Jüngling ertrank so tief.«

Diese »falsche Nonne«, die Widersacherin der Liebenden, wurde nun zu einer »Norne« umgedeutet und somit zu einer germanischen Schicksalsgöttin stilisiert. In den Jahren der NS-Herrschaft erschienen denn auch Schulbücher, in denen explizit erläutert wurde, dass ursprünglich im Liedtext der Ballade von einer »Norne « die Rede gewesen sei und erst der so genannte »Volksmund « dies dann in eine »Nonne « umgesungen habe - eine verblüffend subtile Form der Arisierung der »Königskinder«. Diese Legendenbildung ist indes insofern erfolgreich, als man die Nornen-Version der »Königskinder « und die entsprechende »Ursprungs«-Geschichte dazu bis in die Gegenwart immer wieder antreffen kann. ${ }^{15}$

Die gesamte Geschichte der »Königskinder «-Ballade ist damit natürlich mitnichten erzählt. Doch die beiden angesprochenen Aspekte der VolksliedKonstruktion und Nationalisierung können an einem konkreten Beispiel deutlich machen, wie sich die Konzepte von Volkslied-Idee und-Ideologie in die einzelnen Liedgeschichten einschreiben. Ausgehend von der erörterten Thematik möchte ich abschließend einige Punkte thesenartig ansprechen, die mir für die Arbeit der Popularmusikforschung in diesem Zusammenhang bedenkenswert erscheinen.

15 Dazu ausführlich Eckhard John: »Die >Norne< der >Königskinder<. Zur Genese einer Liedlegende. «In: Historisch-kritisches Liederlexikon (Publ. in Vorber.). 
Popularmusik hat Geschichte. Auch die Popkultur, deren vielfältige Facetten heute meist im Vordergrund stehen, hat eine Vorgeschichte, die älter ist als die Wurzeln von Jazz und Blues, älter auch als die Entwicklung von Schallplatte und Phonograph. Historische Popularliedforschung bietet Möglichkeiten, diese Geschichte kritisch zu rekonstruieren.

Der Begriff des Volksliedes ist dabei allenfalls als historischer Begriff interessant. Darüber hinaus wäre ein Festhalten an dieser Begrifflichkeit für Bemühungen um eine kritische Popularliedforschung jedoch wenig hilfreich. Gegen ihre Verwendung sprechen allein schon die irreführenden Implikationen, die diese Denkfigur in Bezug auf die soziale Trägerschaft freisetzt, erst recht aber ihre ideologischen Aufladungen. Hinzu kommt ein weiterer Aspekt, der es widersinnig erscheinen lässt, dass man den Ausdruck Volkslied einfach als Oberbegriff für die gesamte Breite traditioneller und populärer Lieder nutzbar machen könnte. Denn es ist ein Wesensmerkmal des VolksliedKonzeptes, dass damit immer nur Teilbereiche des populären Liedrepertoires gemeint waren: nämlich jene, die durch die Bezeichnung als »Volkslieder « aufgewertet (sozusagen geadelt) werden sollten (mit entsprechenden Folgewirkungen für deren Bewertung, Pflege, Pädagogisierung etc.). Welche Teilbereiche des Liedrepertoires dies im Einzelnen waren und gegen welche unerwünschten Lieder man die Volkslieder dann jeweils abgrenzte, das war von der jeweiligen Volkslied-Vorstellung abhängig, und das konnte zum Teil sehr unterschiedlich ausfallen. Das Grundmuster der Auswahl und Abgrenzung (gegen unerwünschte Lieder) war dabei jedoch stets eine Konstante.

Auf den Volkslied-Begriff zu verzichten heißt nicht, die Rezeptionsprozesse, die für solche Lieder eine Rolle spielen, aus den Augen zu verlieren. Und dies gilt selbst für das von gänzlich neuen medialen Bedingungen geprägte 20. Jahrhundert: auch hier entwickelten sich populäre Lieder mit weitreichenden Geschichten jenseits der Kulturindustrie, mit gleichwohl hohem Erkennungswert. Für diese Lieder hat die Popularmusikforschung bislang weder ein terminologisch noch theoretisch tragfähiges Konzept.

Das typisch Deutsche am deutschen Volkslied ist allenfalls die deutsche Sprache. Darüber hinaus bieten die Lieder selbst keine erkennbar deutschen Spezifika. Erst die Volkslied-Konzeption und die entsprechenden Sammelintentionen machen das Liedrepertoire »deutsch«. Auch die Idee und Denkfigur vom »Volkslied « ist keine deutsche Erfindung. Sie fand bekanntlich in anderen Sprach- und Kulturräumen ebenso weite Verbreitung. Gleichermaßen ist die Engführung mit nationalistischen Diskursen ein durchaus internationales Phänomen. Und da Typisierungen häufig auf Zuschreibungen von außen beruhen, sollte man sich über einen Punkt keine Illusionen machen: Was auch immer seitens der Forschung als »typisch deutsch« an der 
deutschen Popularmusik angesehen werden mag - für Franzosen, Russen, Schweden, Amerikaner oder Japaner ist völlig klar: das deutsche Volkslied ist typisch deutsch - egal was die Deutschen selbst davon halten.

\title{
Literatur
}

Bausinger, Hermann (2000). Typisch deutsch. Wie deutsch sind die Deutschen? München: C.H. Beck.

Degenhard, Franz Josef (2006). Die Lieder. Berlin: Eulenspiegel.

Hobsbawn, Eric (1983). The Invention of Tradition. Cambridge: Cambridge University Press.

John, Eckhard (1991). »Der Mythos vom Deutschtum in der Musik. Musikwissenschaft und Nationalsozialismus. «In: Die Freiburger Universität in der Zeit des Nationalsozialismus. Hg. v. dems. et al. Freiburg, Würzburg: Plötz, S. 163-190.

John, Eckhard (Hg.) (2006). Die Entdeckung des sozialkritischen Liedes. Münster: Waxmann.

John, Eckhard (2010a). »Das Gedächtnis der Lieder.«In: Vom Minnesang zur Popakademie. Musikkultur in Baden-Württemberg. Hg. v. Badischen Landesmuseum Karlsruhe. Karlsruhe: Badisches Landesmuseum, S. 351-357.

John, Eckhard (2010b). "www.liederlexikon.de: 'Historisch-kritisches Liederlexikon< in the Internet. In: From »Wunderhorn« to the Internet: Perspectives on Conceptions of »Folk Song " and the Editing of Traditional Songs. Trier: WVT, S. 225-233.

Klenke, Dietmar (1998). Der singende »deutsche Mann«. Gesangvereine und deutsches Nationalbewußtsein von Napoleon bis Hitler. Münster: Waxmann.

Weinert, Erich (1970). Gedichte 1919-1925. Hg v. der Deutschen Akademie der Künste in Berlin (= Gesammelte Gedichte 1). Berlin: Aufbau-Verlag.

\begin{abstract}
When we think about national stereotypes in the realm of popular music, the notion of »Volkslieder « (folksongs) is often not far away. Volkslieder seem therefore to function as a natural symbol of this - not only in Germany. In arguing that the invention of the idea of Volkslieder was one of the most successful innovations in the field of popular music, one that has decisively influenced musical history since the end of 18th century, this paper discusses the differences between the idea and ideology of Volkslieder and the actual history of individual songs. The latter is exemplified by the ballad »Es waren zwei Königskinder « (after the ancient story of Hero and Leander). All in all this text pleads for a critical reconstruction of the history of popular music, which is both older than the new technologies of sound reproduction and the roots of jazz or blues.
\end{abstract}

